\title{
Design of an rf quadrupole for Landau damping
}

\author{
K. Papke* and A. Grudiev \\ European Organization for Nuclear Research (CERN), Meyrin 1217, Switzerland
}

(Received 7 March 2017; published 15 August 2017)

\begin{abstract}
The recently proposed superconducting quadrupole resonator for Landau damping in accelerators is subjected to a detailed design study. The optimization process of two different cavity types is presented following the requirements of the High Luminosity Large Hadron Collider (HL-LHC) with the main focus on quadrupolar strength, surface peak fields, and impedance. The lower order and higher order mode (LOM and HOM) spectrum of the optimized cavities is investigated and different approaches for their damping are proposed. On the basis of an example the first two higher order multipole errors are calculated. Likewise on this example the required rf power and optimal external quality factor for the input coupler is derived.
\end{abstract}

DOI: 10.1103/PhysRevAccelBeams.20.082001

\section{INTRODUCTION}

The betatron frequency spread in circular accelerators yield a natural effect of suppressing transverse collective instabilities, the so-called Landau damping [1,2]. The incoherent frequency or tune spread is caused by nonlinearities in the machine. To ensure this mechanism dedicated nonlinear elements, typically octupole magnets, also known as Landau octupoles (LO) are installed in the accelerator [3,4]. However, adiabatic damping and increased beam rigidity reduce their efficiency at higher energies. The Large Hadron Collider (LHC) uses a total of 168 superconducting LOs with a total length of $56 \mathrm{~m}$, which may operate close to their limits after the high luminosity upgrade [5]. Future accelerators may call for more efficient devices in order to satisfy the beam requirements at higher energies.

Recently in [6,7], a superconducting rf quadrupole resonator was proposed as an alternative to the LOs. Its performance is affected likewise by beam rigidity but not adiabatic damping in contrast to magnets, a rf quadrupole resonator introduces a longitudinal instead of a transverse betatron tune spread. In LHC, this is favored by the longitudinal blow-up of the beam. In [8], the stabilization mechanism of a quadrupole resonator has been proven experimentally by using $Q^{\prime \prime}$. Like the quadrupole resonator, $Q^{\prime \prime}$ introduces a longitudinal betatron tune spread in the synchrotron causing Landau damping to stabilize transverse instabilities.

The variation of the betatron frequency due to quadrupolar focusing (so-called detuning) can be split into a

*kai.papke@cern.ch

Published by the American Physical Society under the terms of the Creative Commons Attribution 4.0 International license. Further distribution of this work must maintain attribution to the author(s) and the published article's title, journal citation, and DOI. coherent shift and an incoherent spread where the latter is deciding for Landau damping. Both, the coherent and incoherent parts are proportional to the integrated quadrupolar strength $b_{2}$ which again is correlated to the transverse kick $\Delta \mathbf{p}_{\perp}^{i}$ that a particle $i$ experiences while traversing the cavity along the longitudinal z-axis. In the thin-lens approximation this transverse kick for an ultrarelativistic particle with the charge $q$ is given by:

$$
\Delta \mathbf{p}_{\perp}^{i}=q b_{2}\left(y_{i} \mathbf{e}_{y}-x_{i} \mathbf{e}_{x}\right) \cos \left(\frac{\omega z_{i}}{\beta c_{0}}+\phi_{0}\right),
$$

with $\omega$ as the angular frequency of the quadrupole mode, $\beta$ as the ratio between the particle velocity and the speed of light, the speed of light $c_{0}$ and the rf phase offset $\phi_{0}$. Here, the z-axis coincides with the longitudinal axis of the cavity such that the coordinates $x_{i}$ and $y_{i}$ represent the transverse displacement of the particle from the ideal trajectory, a convention which is used throughout in this paper. The transit time factor is implicitly included in (1) via $b_{2}$. Figure 1 shows the principle of two different quadrupole resonators using a transverse magnetic (TM) or transverse electric (TE) mode, respectively.

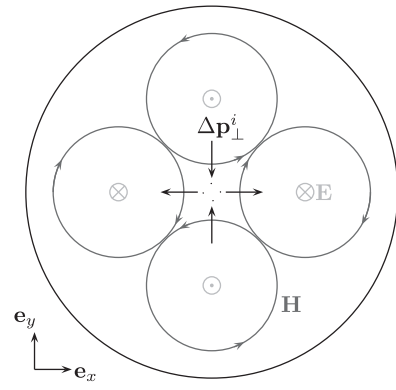

(a)

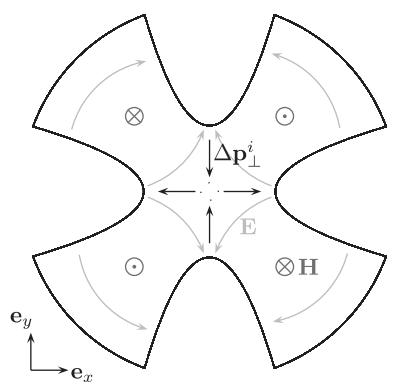

(b)
FIG. 1. Cross section of quadrupolar field profiles providing the transverse kick to the particle according to (1). The force directions corresponds to a negative charge $q$. (a) Pillbox with TM-type mode. (b) Four-Vane-Cavity with TE-type mode. 
In [6], it has been demonstrated that a rf quadrupole resonator can result in a significantly more compact solution than a comparable set of octupole magnets providing the same tune spread. In this paper we present the first detailed design and optimization studies of a rf quadrupole resonator for Landau damping. Although obtained on the basis of the HL-LHC, many results presented here are generic and can be applied or adapted to other machines. As for an accelerating cavity, the choice of the geometry determines nearly all basic parameters, such as quadrupolar strength $b_{2}$, peak electric and magnetic field $\left(E_{p k}\right.$ and $\left.B_{p k}\right)$, the longitudinal and transverse impedance as well as multipacting barriers. The latter may limit the maximum achievable $b_{2}$ if the geometry provides so-called hard barriers or increase the conditioning time in case of soft barriers. Usually, multipacting is investigated for each cavity individually and, in general, cannot be predicted analytically. In consideration of the numerous cavities presented in this paper, we will not treat this part of the cavity design.

Due to the wide experience in the accelerator community, the author wants to focus first on elliptical cavities (Sec. III). The results serve as reference for a more compact geometry presented in Sec. IV whose shape was motivated by the classical RFQ four vane geometry used in proton linacs. Likewise in Secs. III and IV, the lower and higher order mode spectrum for each cavity type is discussed and possible damping approaches are proposed. In Sec. V the required input power for a cavity is derived in order to compensate the beam loading. Along the cavity design we propose different approaches of optimization based on the figures of merit such as $b_{2} / B_{p k}$ introduced in the following section.

\section{CAVITY DESIGN PARAMETER}

The parameters used to optimize the SC cavity can be differentiated into two categories. First, parameters that are derived from eigenmode simulations such as the quadrupolar strength $b_{2}$ and second, parameters derived from wakefield simulations such as the transverse and longitudinal impedance. The latter is considerably more time-consuming than eigenmode calculations, hence, it is desirable to minimize the number of wakefield simulations. We propose, first, to optimize the designs independently of the impedance but with varying aperture which has the main influence on the impedance and, second, to calculate the impedance as a subsequent step for each preoptimized design. In the following, we discuss all the design parameters involved in the optimization.

\section{A. Integrated quadrupolar strength}

The integrated quadrupolar strength $b_{2}$ is the prime figure of merit and should be as high as possible in order to minimize the total number of cavities providing a certain betatron frequency spread. It is understood as the maximum transverse momentum a quadrupolar mode is able to provide to a probe charge during its passage. In contrast to magnets the transverse force in a cavity is rf modulated. In order to give a quantity for beam dynamic simulation comparable to the focusing strength of magnets we integrate the force or the field over $z$. In the following, we denote the quantity simply as quadrupolar strength $b_{2}$ keeping in mind the longitudinal integration. Generally, the electromagnetic field yielding a transverse Lorentz Force $\mathbf{F}_{\perp}$ can be decomposed into multipoles $[9,10]$. Using cylindrical coordinates, the $\rho$-component of the transverse impulse can be decomposed as follows:

$$
\begin{aligned}
\Delta \mathbf{p}_{\perp} \cdot \mathbf{e}_{\rho} & =\frac{1}{c_{0}} \int_{0}^{L} \mathbf{F}_{\perp} \cdot \mathbf{e}_{\rho} d z \\
& =q \sum_{n=1}^{\infty} \rho^{n-1}\left[a_{n} \sin (n \varphi)+b_{n} \cos (n \varphi)\right],
\end{aligned}
$$

We define $\varphi=0$ as the azimuthal position where the transverse momentum provided by the quadrupole mode is maximal, hence, it corresponds to either the $\mathrm{x}$ - or $\mathrm{y}$-axis in (1). Following this convention, the coefficients $a_{n}$ can be assumed zero. An ideal quadrupole resonator gives only a nonzero value for the term $b_{2}$, however, geometries which are not axial symmetric, typically, exhibit higher order terms as discussed in Sec. IV E. The coefficients $b_{n}$ can be calculated via the radial Lorentz force $F_{\rho}$ taking into account the transit time of the particle:

$b_{n}=\frac{1}{q c_{0}} \frac{1}{\pi} \int_{-\pi}^{\pi} \frac{\cos (n \varphi)}{\rho^{n-1}} \int_{0}^{L} F_{\rho} \exp \left(j \frac{\omega z}{\beta c_{0}}\right) d z d \varphi$

or by utilization of the Panofsky-Wenzel theorem [11] via the z-component of the electric field $E_{z}$ instead of $F_{\rho}$ :

$$
b_{n}=\frac{j n}{\omega} \frac{1}{\pi} \int_{-\pi}^{\pi} \frac{\cos (n \varphi)}{\rho^{n}} \int_{0}^{L} E_{z} \exp \left(j \frac{\omega z}{\beta c_{0}}\right) d z d \varphi .
$$

Applied on numerically obtained eigenmode solutions, the first option has shown to be more precise for higher order terms while the second option is easier and faster to evaluate. In Table I, quadrupolar strengths are shown providing an equivalent stabilization effect as the LOs in HL-LHC at a specific chromaticity [12]. The values are based on macro particle tracking simulation comprising $6 \times 10^{5}$ turns. Though only for a specific case of instability, we use these values as a reference for the cavity design throughout in this paper.

The maximum coherent tune shift the quadrupole resonator is able to stabilize by Landau damping is dependent on the frequency as the curvature of the cosine wave along the bunch introduce the nonlinearity. Practically, the 
TABLE I. Required $b_{2}$ yielding the same stabilization as provided by LOs for HL-LHC at a chromaticity $Q_{x, y}^{\prime}=10$ [12].

\begin{tabular}{lc}
\hline \hline Frequency $[\mathrm{MHz}]$ & $b_{2}[\mathrm{Tm} / \mathrm{m}]$ \\
\hline 400 & 0.35 \\
800 & 0.27 \\
1200 & 0.40 \\
\hline \hline
\end{tabular}

frequency is limited to harmonics of the main rf system. In case of LHC we can use either 400, 800, or $1200 \mathrm{MHz}$ with respect to cavity size and difficulty of fabrication. According to the results shown in Table I a frequency of $800 \mathrm{MHz}$ is favored which is consistent with frequency considerations presented in [6].

As for accelerating cavities, the strength is limited by either the peak magnetic or electric surface field. Therefore, it is reasonable to relate the integrated quadrupolar strength always to these quantities according to: $b_{2} / B_{p k}$ and $b_{2} / E_{p k}$.

Finally, the relation between the quadrupolar strength and the longitudinal or transverse (kick) voltage, respectively, are considered. In cylindrical coordinates, the longitudinal voltage $V_{\|}$is defined as:

$$
V_{\|}(\rho, \varphi)=\int_{0}^{L} E_{z}(\rho, \varphi, z) \exp \left(j \frac{\omega z}{\beta c_{0}}\right) d z .
$$

In the first order, the azimuthal dependency of the quadrupolar $E_{z}$-component can be described by a $\cos (2 \varphi)$ term $^{1}$ which simplifies $(2 b)$ to:

$$
b_{2}=j \frac{2}{\omega} \frac{1}{\rho_{0}^{2}} V_{\|}\left(\rho_{0}, 0\right)
$$

with $V_{\|}$proportional to $\rho^{2}$ [13]. The longitudinal voltage evaluated at $\varphi=0$ yield its magnitude in the azimuthal direction. Using the Panofsky-Wenzel theorem [11], we obtain an expression for the transverse voltage:

$$
V_{\perp}=-j c_{0} / \omega \nabla_{\rho, \varphi} V_{\|},
$$

which can be understood as either a radial or azimuthal kick voltage and is proportional to $\rho$. We further obtain the relation between the quadrupolar strength and the maximum transverse kick voltage by partial differentiation of (4) and substitution with (5):

$$
b_{2}=-\frac{1}{c_{0}} \frac{1}{\rho_{0}} V_{\perp}\left(\rho_{0}, 0\right) .
$$

The latter relation underlines the physical meaning of $b_{2}$ as the maximum transverse momentum the quadrupole mode is able to provide to a particle. Equations (4) and (6) can be

\footnotetext{
${ }^{1}$ The $\cos (2 \varphi)$ dependency is also very accurate for the fourvane cavity as simulations have shown.
}

used to calculate the required rf power for the cavity based on the quadrupolar strength $b_{2}$ (Sec. V).

\section{B. Field limitation and losses}

The ratio between the surface peak fields $B_{p k} / E_{p k}$ is another important aspect for designing superconducting cavities. The effect of high electric and high magnetic surface field is very different. While the first usually causes field emission, the latter may locally heat up the material above the critical temperature and induce the cavity to quench. It is desirable to balance the peak fields according to technological limits, hence, not to be limited by only one field type. We have chosen the superconducting TESLA cavity [14] as a reference with $B_{p k} / E_{p k}=2.13 \mathrm{mT} /(\mathrm{MV} / \mathrm{m})$ which is close to assumptions made in [15] for the rf-dipole crabbing cavity.

To minimize the losses in accelerating cavities typically the product of the geometry factor $G$ and the $(R / Q)$ value is considered to be maximized [16]. This is equivalent to the product of shunt impedance and surface resistance. A similar definition is applied in [15] for deflecting type cavities by using the transverse voltage $V_{\perp}$ and $(R / Q)_{\perp}$ of a dipole mode. Analogously, we may introduce the quantity $G \cdot b_{2}^{2} /(\omega U)$ to describe the cavity losses in a quadrupole resonator with the stored energy $U$. However, due to the low rf power needed for this cavity (Sec. V), we are less concerned with the overall surface loss, but rather more with peak fields.

\section{Impedance and HOM damping}

The limited impedance budget guaranteeing a stable beam operation in the accelerator asks for a minimized narrow and broadband impedance of all additional elements in a synchrotron such as aperture transitions, gate valves, beam collimators, et cetera. We use the definition from [17] to quantify the effective longitudinal and transverse impedance of the quadrupole resonator denoted as $\left(Z_{\|} / n\right)_{\text {eff }}$ and $\left(Z_{\perp}\right)_{\text {eff }}$ :

$$
\begin{aligned}
\left(Z_{\|} / n\right)_{\mathrm{eff}} & =\frac{\int_{0}^{\infty} Z_{\|}(\omega) \frac{\omega_{0}}{\omega} h_{1}(\omega) d \omega}{\int_{0}^{\infty} h_{1}(\omega) d \omega}, \\
\left(Z_{\perp}\right)_{\mathrm{eff}} & =\frac{\int_{0}^{\infty} Z_{\perp}(\omega) h_{0}(\omega) d \omega}{\int_{0}^{\infty} h_{0}(\omega) d \omega},
\end{aligned}
$$

with $\omega_{0}$ as the angular revolution frequency around the circular accelerator and the bunch spectrum $h_{1}$ given by $h_{n}=\left(\omega \sigma_{z} / c_{0}\right)^{2 n} \exp \left[-\left(\omega \sigma_{z} / c_{0}\right)^{2}\right] / \Gamma(n+0.5)$ where $\sigma_{z}$ corresponds to the RMS bunch length. A Gaussian distribution is assumed here. ${ }^{2}$ The longitudinal and transverse

\footnotetext{
${ }^{2} \mathrm{LHC}$ operates with a revolution frequency of $f_{0}=11.254 \mathrm{kHz}$. All impedance results presented in this paper are calculated for a bunch length with RMS sigma of $\sigma_{z}=80 \mathrm{~mm}$.
} 
TABLE II. Impedance budget for LHC [17].

\begin{tabular}{ll}
\hline \hline Parameter & Value \\
\hline$\left(Z_{\|} / n\right)_{\text {eff }}$ & $93 \mathrm{~m} \Omega$ \\
$\left(Z_{\perp}\right)_{\text {eff }}$ & $20 \mathrm{M} \Omega$ \\
\hline \hline
\end{tabular}

impedances $Z_{\|}$and $Z_{\perp}$ can be derived from wakefield simulations using e.g. ABCI [18], ECHO(2D)[19] or CST PARTICLE STUDIO ${ }^{\circledR}$ (CST PS) [20]. The total effective impedance of the required quadrupole resonators for LHC should be far below the limits shown in Table II. The impedance is dominated by the aperture and tapering of the beam pipe. In general, the smaller the aperture the higher the longitudinal and transverse impedance.

Furthermore, the choice of aperture or beam pipe radius should take into account the extraction of lower order and higher order modes (LOM and HOM). Trapped modes with high $(R / Q)$ values may require additional couplers or waveguides close to the cavity whereas propagating modes may be damped even outside of the cryomodule by lossy material in the beam pipe. We will consider different options for each cavity type.

\section{Mechanical restrictions}

Finally, a few mechanical limitations should be mentioned, they originated from the manufacturing procedures and space limitation: (i) The minimal aperture is $20 \mathrm{~mm}$. (ii) Due to the process of bending of niobium sheet when forming a cavity, the minimal curvature radius of a cavity wall is $10 \mathrm{~mm}$. (iii) The maximum length of the entire system including the cryomodule(s) is $10 \mathrm{~m}$ (LHC layout constraint).

\section{ELLIPTICAL CAVITY}

As it is widely used in the SRF community, the standard non-reentrant elliptic cavity provides a good reference for other cavity types which are typically more complicated to fabricate. The cavity profile is defined by two conjugated elliptic arcs connected with a straight part [Fig. 2(a)]. Corresponding to their location, the first ellipse defines the contour of the equator whereas the second one describes the iris contour. Hence, we denote appropriate parameters with the subscripts "eq" and "ir". In the frame of the optimizations, we observed a correlation between the optimal transition angle $\alpha$ (common tangential angle of both ellipses and the straight part) and the quadrupolar strength $b_{2}$ that motivated the extension of the investigations towards reentrant profiles [Fig. 2(b)]. Indeed, depending on the iris radius $r_{\text {ir }}$, either a non-reentrant $(\alpha<90 \mathrm{deg})$ or a reentrant $(\alpha>90 \mathrm{deg})$ elliptical cavity provides the higher transverse kick, both assumed to be optimized with respect to the quadrupolar strength.

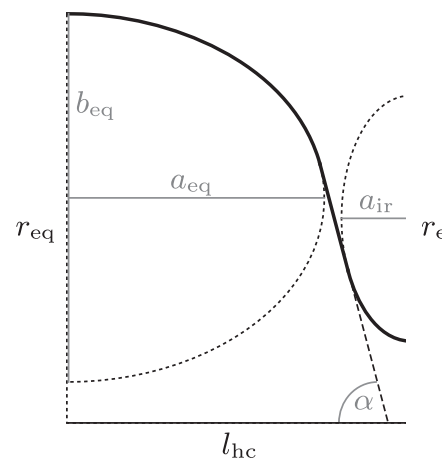

(a)

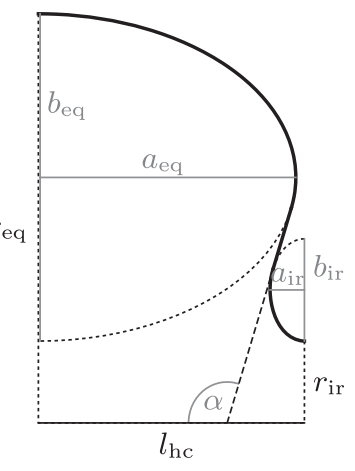

(b)
FIG. 2. Profile of elliptical half cells with equator radius $r_{\mathrm{eq}}$, iris radius $r_{\mathrm{ir}}$ and length $l_{\mathrm{hc}}$. The dotted lines subscribe the corresponding ellipses as well as the connecting straight with the concerted tangential angle $\alpha$. (a) Non-reentrant cavity with $\alpha<90 \mathrm{deg}$ and (b) reentrant cavity with $\alpha>90 \mathrm{deg}$.

In analogy to the analytic eigenmode solutions of a cylindrical pillbox, it is obvious to choose either the $\mathrm{TM}_{210}$ or the $\mathrm{TE}_{211}$ mode, both, resulting in the quadrupolar field distributions as qualitatively shown in Fig. 1. However, the latter one achieves approximately $40 \%$ less quadrupolar strength for the same amount of stored energy in the cavity due to its longitudinal dependency. Therefore we focus our considerations on the $\mathrm{TM}_{210}$ (Fig. 3). Before we discuss the results of the final cavities and conclude for the HL-LHC, we present an efficient optimization process which can be applied to any other cavity design that is axially symmetric.

\section{A. Optimization}

The prime objective that defines the "best" cavity shape is given by the maximum possible quadrupolar strength $b_{2}$ with respect to the limiting surface peak fields (either $B_{p k}$ or $\left.E_{p k}\right)$. Moreover, the optimization includes a further condition given by a fixed frequency. Typically, the

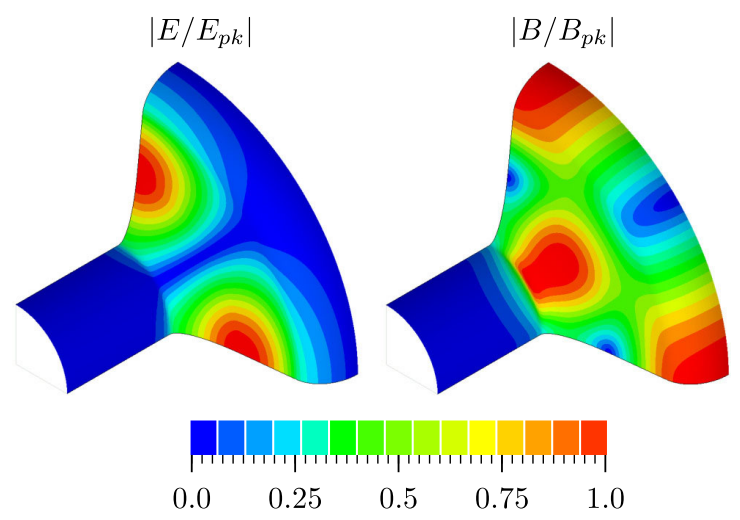

FIG. 3. Normalized fields distribution of the $\mathrm{TM}_{210}$ mode on the surface of an elliptical cavity section. 
frequency can be adjusted via the equator radius $r_{\text {eq }}$. As an example, Fig. 4 shows the properties of various elliptical cavities which differ in length $h_{\mathrm{hc}}$ and equator ellipse, all adjusted to a frequency of $800 \mathrm{MHz}$. The results show a limitation by the magnetic field $\left(B_{p k} / E_{p k}>2.13\right)$ which turned out to be the case throughout for all considered elliptical cavities operating in $\mathrm{TM}_{210}$ mode. The maximum in Fig. 4 appears approximately at a cavity length of half the wave length $\lambda$. In many cases (especially for the nonreentrant cavities with apertures $r_{\text {ir }}>70 \mathrm{~mm}$ ), a significant field contribution appears still at $\lambda / 2$ resulting in a larger optimal cavity length.

Like the equator radius $r_{\text {eq }}$ due to the fixed frequency, we can exclude the iris radius as well from the optimization because the quadrupolar strength together with the longitudinal and transverse impedance monotonously increase with decreasing cavity aperture. Instead, we optimize several designs with varying iris radius and subsequently investigate their impedance with respect to the maximum achievable quadrupolar strength per cavity.

The remaining free parameter for the optimization beside the cavity length described by $l_{\mathrm{hc}}$ are the half axes

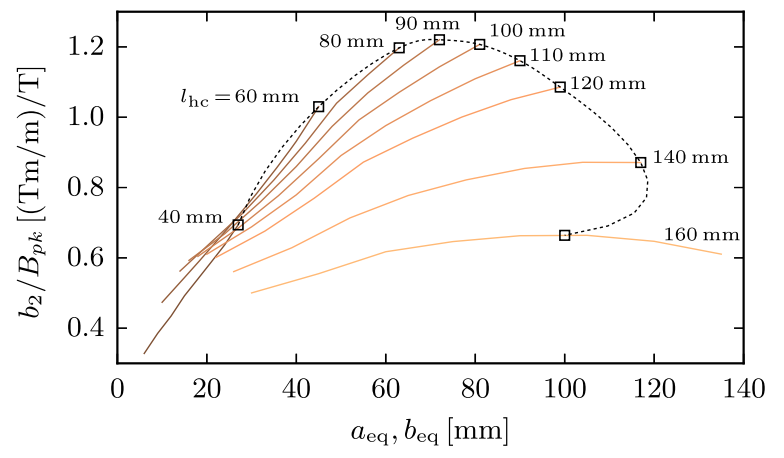

(a)

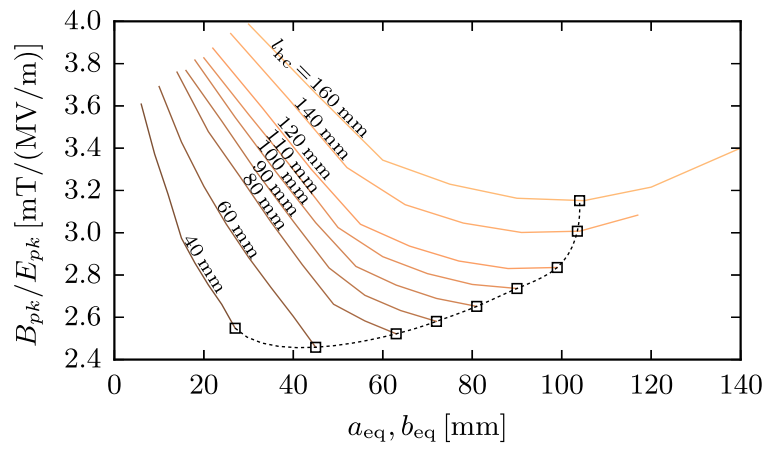

(b)

FIG. 4. Quadrupolar strength $b_{2}$ with respect to the surface peak field $B_{p k}$ (a) and the ratio $B_{p k} / E_{p k}$ (b) of an $800 \mathrm{MHz}$ non-reentrant elliptical cavity. The iris ellipse is fixed to $a_{\mathrm{ir}}=$ $b_{\text {ir }}=10 \mathrm{~mm}$ while the equator ellipse varies under the condition $a_{\text {eq }}=b_{\text {eq }}$ resulting in a circular profile. Besides, different cavity lengths $l_{\mathrm{hc}}$ are compared to each other showing the maximum in (a) close to $\lambda / 2$.

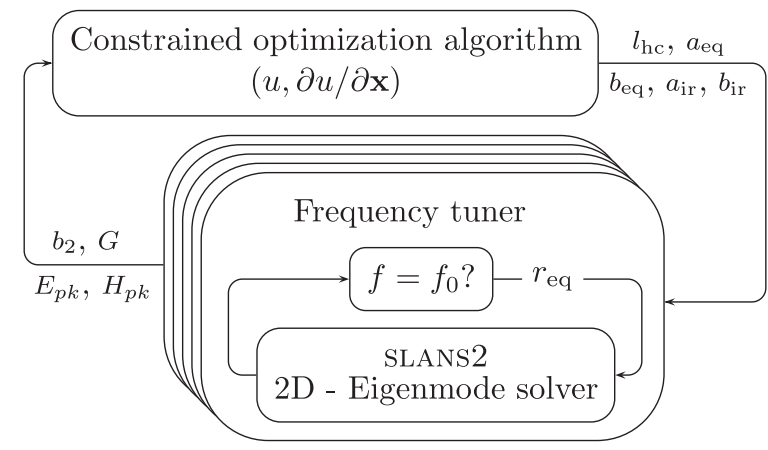

FIG. 5. Sketch of the cavity optimization. The optimizer minimizes the quality function $u(\mathbf{x})=B_{p k} / b_{2}$ where $\mathbf{x}$ is a vector of free design variables such as $a_{\mathrm{ir}}, b_{\mathrm{ir}}, a_{\mathrm{eq}}, a_{\mathrm{eq}}$ and $l_{\mathrm{hc}}$.

of the iris and equator ellipses $\left(a_{\mathrm{ir}}, b_{\mathrm{ir}}, a_{\mathrm{eq}}\right.$, and $\left.a_{\mathrm{eq}}\right)$. Note, the tangential angle $\alpha$ depends on the other parameters shown in Fig. 2. We have chosen SLANS2 [21] as eigenmode solver which calculates multipole modes in axially symmetric rf cavities using the continuous Galerkin method applied on quadrangle bi-quadratic elements. Several tests depicted a similar or higher field accuracy than achieved by CST [20] or HFSS [22] with comparable element size. However, the calculation is significantly faster as the eigenmode problem is reduced to a two-dimensional problem in the $(\rho, z)$-plane. Furthermore, the evaluation of the quadrupolar strength simplifies to an integral in $z$-direction, only, as the integral $\int d \varphi$ result in the factor $\pi$.

A wrapper around SLANS2 has been developed in PYTHON in order to automatize the process of finding the "best" shape to a large extend (Fig. 5). It consist of an optimizer that creates and distributes elliptical designs over a number of parallel running tuners used for the frequency adjustment. The optimizer uses a constrained BFGS algorithm ${ }^{3}$ to minimize an arbitrary chosen quality function $u(\mathbf{x})$ with the special feature that the entries of the Jacobian $\partial u / \partial x_{i}$ (with $x_{i}$ as free design variables) are calculated in parallel. Using a five-point differentiation in each variable the parallelization reduces the evaluation by a factor of 10 on an Intel i7 processor of the third generation. Depending on the cavity size, an optimization of five parameter was finished within between half a day and three days. In principle, it is possible to extend the scheme on more than five parameters, however, one may consider a simpler differentiation rule or a processor with more cores.

\section{B. Optimal design parameters}

The optimizations have been carried out with varying iris radii $r_{\text {ir }}$ between $25-60 \mathrm{~mm}$ for the reentrant cavities and

\footnotetext{
${ }^{3}$ Broyden Fletcher Goldfarb Shanno algorithm, a group of hill-climbing optimization techniques.
} 
between $50-175 \mathrm{~mm}$ for the non-reentrant cavities, a total of 22 designs (See Supplemental Material at [23] for detailed results).

From Fig. 6, it can be seen that the transition between reentrant and non-reentrant cavities appears at an aperture of approximately $r_{\mathrm{ir}}=60 \mathrm{~mm}$. Hence, depending on the iris radius either the first (if $r_{\text {ir }} \leq 60 \mathrm{~mm}$ ) or the second cavity type (if $r_{\text {ir }}>60 \mathrm{~mm}$ ) potentially perform better in terms of $b_{2} / B_{p k}$.

The optimal cavity length is always close to half of the wavelength if the iris ellipse is small compared to the equator ellipse [Figs. 7(a) and 7(b)]. However the optimization tends towards larger iris ellipses the greater the aperture. For mechanical and economic reasons, we have to restrict the ratio $a_{\mathrm{ir}} / a_{\mathrm{eq}}$. The upper limit of 1.0 avoids too large iris ellipses that artificially lengthen the cavity whereas the lower limit at 0.1 avoids too small curvatures and a too high electric surface field at the iris.

The remaining design parameters can be expressed by the ellipticity of iris and equator which we define as the logarithmic ratio of the vertical and horizontal half axis of an ellipse [Figs. 7(c) and 7(d)].

In case of the non-reentrant cavity, the ellipticity increases (equator) or decreases (iris) approximately linearly with the aperture. Note, the logarithmic definition yield an exponential dependency of the ratio $b_{\mathrm{x}} / a_{\mathrm{x}}$. In a first iteration the optimized designs for iris radii $r_{\text {ir }} \leq$ $80 \mathrm{~mm}$ provided throughout an almost circular contour [data set with dashed lines in Fig. 7(c)]. In a second iteration a lower constraint was applied ensuring at least a three times larger vertical than horizontal axis. This improved slightly the performance of the corresponding cavities and yield a more continuous correlation between the relative quadrupolar strength $b_{2} / B_{p k}$ and the iris radius $r_{\mathrm{ri}}$.

Toward larger beam pipe radii $r_{\text {ir }}>90 \mathrm{~mm}$ the minimum of the goal function is very shallow, meaning that any small parameter change especially in the ellipticities will not have a notable effect on the $b_{2}$.

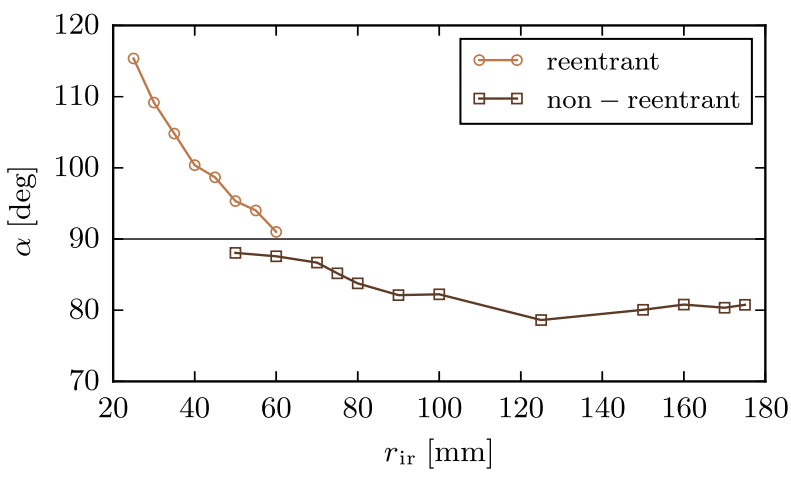

FIG. 6. Optimal tangential angle as a function of the iris radius $r_{\text {ir }}$. The transition between a reentrant and non-reentrant elliptical cavity is at $r_{\mathrm{ir}} \sim 60 \mathrm{~mm}$.

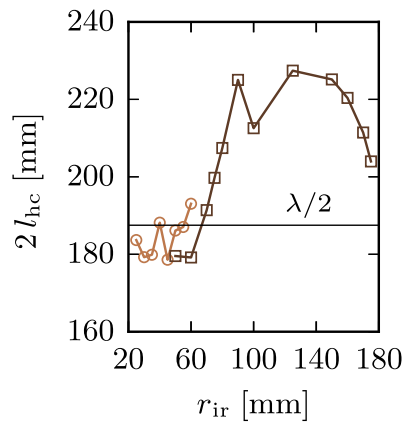

(a)

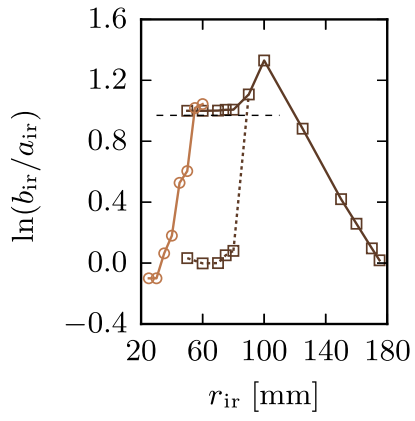

(c)

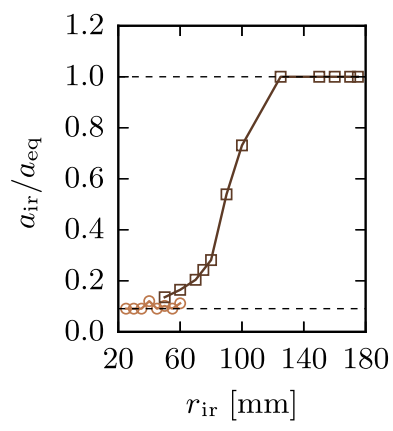

(b)

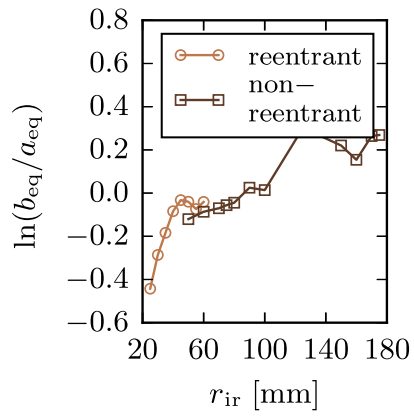

(d)
FIG. 7. Optimized cavity parameters as functions of the iris radius $r_{\text {ir }}$. (a) Cavity length. (b) Ratio between iris and equator ellipse by means of their horizontal half axes. Due to mechanical constraints, this ratio is limited. (c) - (d) Logarithmic ratio between the vertical and horizontal half axis (ellipticity) for the iris and equator ellipse.

\section{Properties of the optimized cavities}

The properties of the designs are summarized in Figs. 8 and 9 as functions of the iris radius. Each abscissa point is related to an optimized geometry. Due to the smaller apertures the maximal achievable quadrupolar strength of reentrant elliptical cavities is higher than that

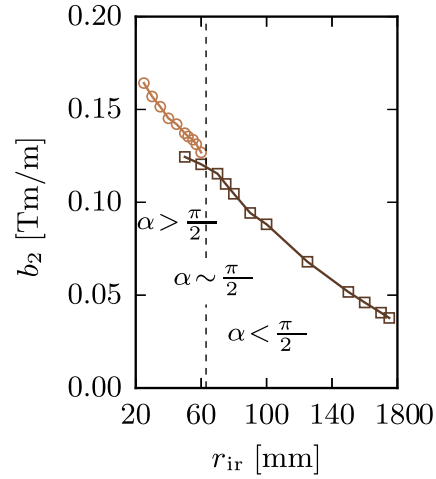

(a)

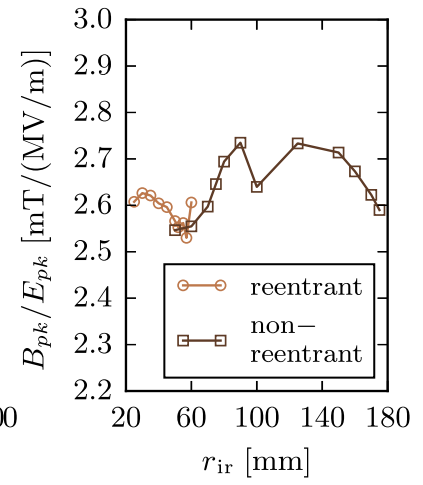

(b)
FIG. 8. (a) Maximal quadrupolar strength of the optimized cavities distinguished by their aperture assuming a magnetic peak field at the surface of $B_{p k}=100 \mathrm{mT}$. (b) The corresponding ratio of the surface peak fields $B_{p k} / E_{p k}$. 


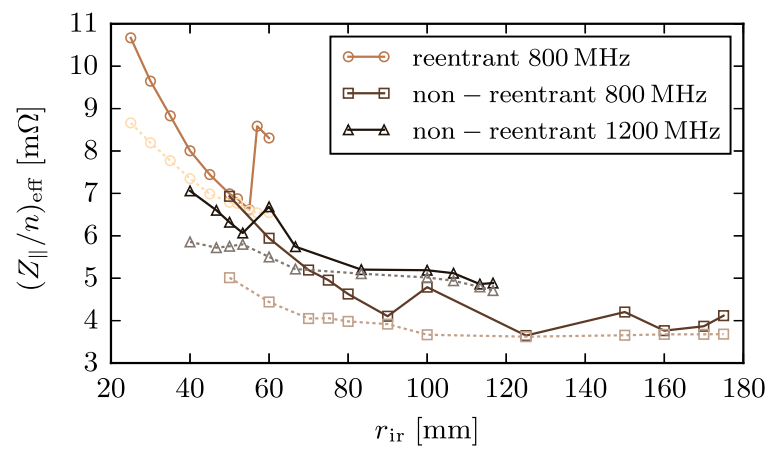

(a)

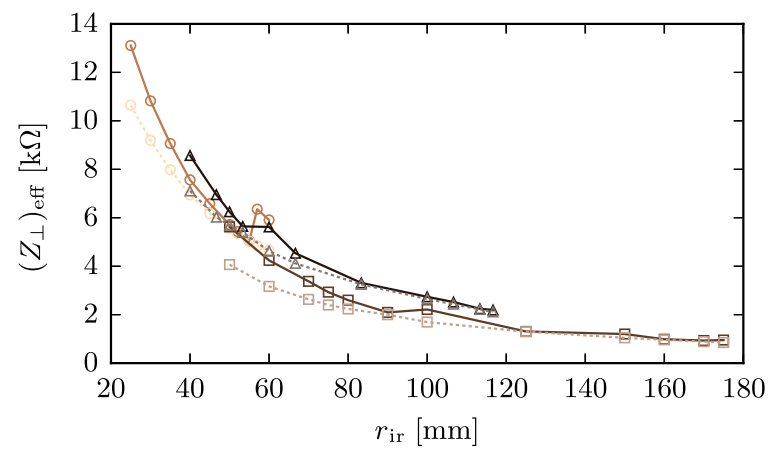

(b)

FIG. 9. Effective longitudinal (a) and transverse (b) impedance accumulated over the number of required cavities to satisfy the conditions in Table I. In solid, the number of cavities is rounded up. In dashed, the number of cavities is not rounded providing smoother relation that shows a shallow minimum at $r_{\mathrm{ir}}=125 \mathrm{~mm}$ for the longitudinal impedance.

of non-reentrant elliptical cavities. The first are limited to around $b_{2}=0.16 \mathrm{Tm} / \mathrm{m}$ with an aperture of $r_{\text {ir }}=25 \mathrm{~mm}$ while the latter achieve a maximum of $b_{2}=0.12 \mathrm{Tm} / \mathrm{m}$ with apertures between $r_{\text {ir }}=50-60 \mathrm{~mm}$. The ratio of the surface peak fields is consistently around $2.6 \mathrm{mT} /(\mathrm{MV} / \mathrm{m})$ as shown in Fig. 8(b). The limitation is clearly determined by the magnetic field at the surface.

With increasing aperture the number of required cavities to provide the same betatron tune spread increases monotonically, and thereby the length and impedance of the whole system. Due to a limited space of $10 \mathrm{~m}$ length for HL-LHC, the iris radius of the elliptical cavity must not be greater than $r_{\text {ir }}=125 \mathrm{~mm}$.

A system of non-reentrant $800 \mathrm{MHz}$ elliptical cavities that satisfy the requirements of Table I provides an effective longitudinal impedance of at least $4 \mathrm{~m} \Omega$, almost $5 \%$ of the entire LHC impedance budget (Fig. 9). This value can be significantly larger in a system that should provide a large margin with respect to the numerous kinds of instabilities in a synchrotron. Reentrant cavities, operating at the same frequency provide more than $8 \%$ of the budget, which is a clear disadvantage of this cavity type besides. A rescaled and more compact version consisting of nonreentrant cavities operating at $1200 \mathrm{MHz}$ provides a total
TABLE III. Trapped lower and higher order modes for a $800 \mathrm{MHz}$ non-reentrant elliptical cavity with $r_{\mathrm{ir}}=90 \mathrm{~mm}$.

\begin{tabular}{lcrrr}
\hline \hline \multicolumn{2}{c}{ Monopole modes } & & \multicolumn{2}{c}{ Dipole modes } \\
\cline { 1 - 2 } \cline { 5 - 5 } Mode & Frequency [MHz] & & Mode & Frequency [MHz] \\
\hline $\mathrm{TM}_{010}$ & 417.7 & & $\mathrm{TM}_{110}$ & 594.5 \\
$\mathrm{TM}_{020}$ & 896.2 & & $\mathrm{TE}_{111}$ & 778.2 \\
$\mathrm{TE}_{011}$ & 1107.5 & & $\mathrm{TE}_{121}$ & 1281.0 \\
$\mathrm{TM}_{011}$ & 1137.9 & & $\mathrm{TM}_{131}$ & 1586.7 \\
$\mathrm{TE}_{021}$ & 1430.9 & & & \\
\hline \hline
\end{tabular}

effective longitudinal impedance which lies between 4 and $6 \%$ of the budget. The effective transverse impedance [Fig. 9(b)] is not of concern since the results are throughout small against the defined impedance budget in Table II.

To conclude, one may chose for HL-LHC a nonreentrant elliptical cavity with $r_{\text {ir }}=90-100 \mathrm{~mm}$. The required three cavities result in nearly the lowest possible effective longitudinal impedance of $4 \mathrm{~m} \Omega$.

\section{LOMs and HOMs}

In analogy to the cylindrical pillbox whose eigenmode solutions can be calculated analytically, we have to deal with monopole and dipole modes below the operating frequency of the quadrupolar mode. All of them are trapped inside the cavity and potentially excited by the beam. Moreover higher order modes appear up to $1.7 \mathrm{GHz}$ in the longitudinal and transverse wake spectra. A summary can be found in Table III for an optimized cavity with $r_{\text {ir }}=90 \mathrm{~mm}$.

Taking advantage of the field distribution of the quadrupole mode it is reasonable to extract the power of LOMs and HOMs by placing couplers around the equator at the low field regions of the operating mode (Fig. 10). This has the advantage that the coupler does not require a

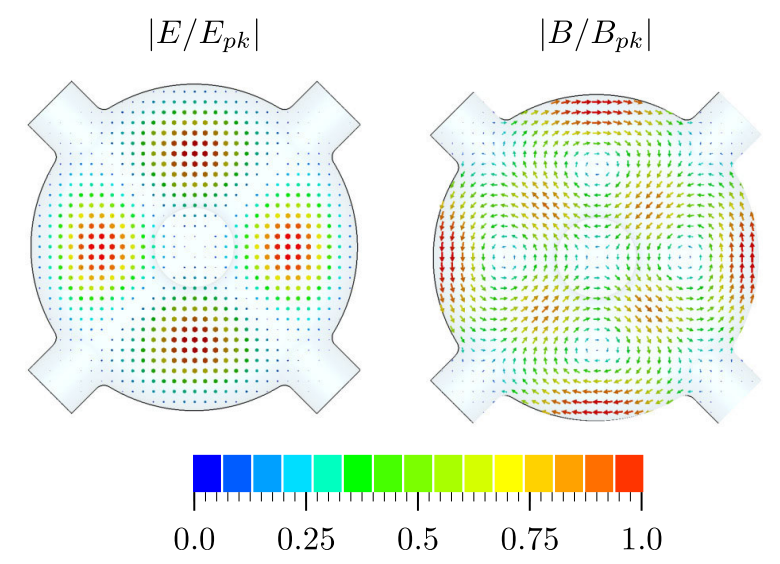

FIG. 10. Electric and magnetic field distribution of the quadrupole mode in the transverse plane. The cavity is equipped with ports at the low field regions potentially suitable for damping lower and higher order modes. 
notch filter at $800 \mathrm{MHz}$ or at least not a filter with so strict requirements as typically for HOM couplers of accelerating type cavities. Furthermore, the ports yield a significant frequency separation between the two polarizations of the quadrupole mode in the order of 10-20 MHz. To excite the correct polarization, a special coupler design is required. Preliminary simulations have shown that the ports can have a diameter up to $100 \mathrm{~mm}$ without notably modifying the field distribution or enhancing the surface field.

\section{FOUR-VANE CAVITY}

The disadvantages of the previously discussed elliptical cavities can be summarized as follows: (i) The maximum achievable quadrupolar strength $b_{2}$ per cavity is relatively low which result in a large number of cavities. (ii) The large size of the cavity with an equator radius of approximately $300 \mathrm{~mm}$ and a length of around $200 \mathrm{~mm}$ requires correspondingly large cryomodules, hence, more cooling power is needed. All this increases the cost of the entire system. (iii) The limitation by the magnetic field on the surface may cause the cavity to quench. (iv) The effective longitudinal impedance is high with respect to the LHC impedance budget. All these drawbacks can be resolved by a cavity design whose contour in the transverse plane forms a quadrupole-like electric field according to the boundary conditions. In principle, such a geometry is given in Fig. 1(b). If carefully chosen, the field along the transverse contour (surface field) can be more "evenly" distributed than in case of the elliptical cavity (compare Fig. 10) which allows a higher quadrupolar strength within the limits of the surface fields. The real cavity is obtained by a smooth transition from the quadrupolar contour to the beam pipe. It should be smooth in order to avoid field enhancements and to reduce the longitudinal and transverse impedance. Figure 11 illustrates the cavity design which we denote as four-vane cavity. It is obvious that the disadvantages mentioned above can be only overcome at the cost of a more complex design.

Similar as for the elliptical cavity we define the transverse contour by two conjugated arcs connected with a straight line. However, the equator is simplified to a circle with the radius $a_{\text {eq }}$. Nevertheless, we will continue with the previous notation and talk about iris and equator ellipse to avoid any confusion with the equator radius $r_{\mathrm{eq}}$. The profiles which are shown in Figs. 11(c) and 11(d) have to be mirrored in the azimuthal direction seven times to obtain the complete transverse contour as the one sketched in Fig. 1(b).

Since the arc of the equator ellipse is located in the region of high magnetic fields (Fig. 12), we require that the radius $a_{\mathrm{eq}}$ to be as large as possible, which has the convenient side effect of less parameters to be optimized. Depending on the iris radius $r_{\text {ir }}$ and iris ellipse $\left(a_{\text {ir }}, b_{\text {ir }}\right)$, the center of the equator ellipse is either on the diagonal of the

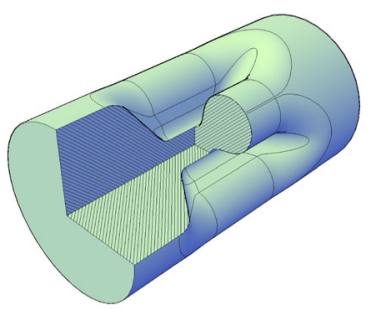

(a)

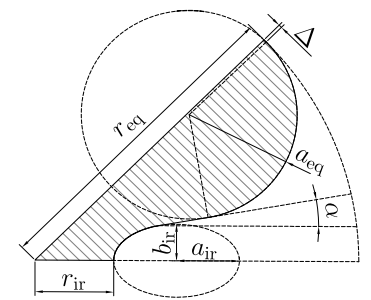

(c)

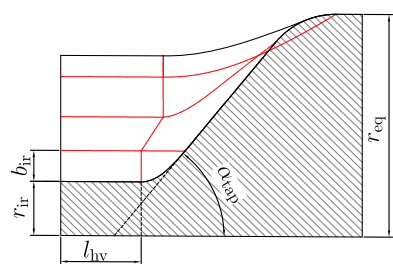

(b)

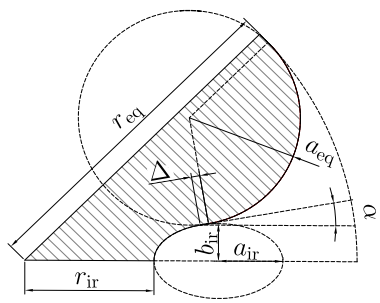

(d)
FIG. 11. Four-vane cavity design. (a) 3D view of the cavity with an open-left segment to depict the unique parts of the longitudinal and transverse cross-section. A quarter of the longitudinal cross section is shown in (b) while (c) and (d) show two different versions of the transverse cross section (only an eighth). The difference is given by a displacement of the circle resulting from a larger iris radius $r_{\mathrm{ir}}$ in (d). The beam is located along the bottom line in (b) or at the lower left corner in (c) and (d), respectively.

length $r_{\text {eq }}$ [Fig. 11(c)] or displaced from this diagonal [Fig. 11(d)]. In the latter case, the length of the straight between the conjugated arcs is zero. However, in the view of the numerous eigenmode simulations it is recommended to insert additional but very short straight segments in the contour to avoid construction or meshing problems within the simulation tools. In Fig. 11, these parts are labeled by the symbol $\Delta$.

The tangential angle $\alpha$ is limited to a positive range between 0 and $20 \mathrm{deg}$. A reentrant profile with negative

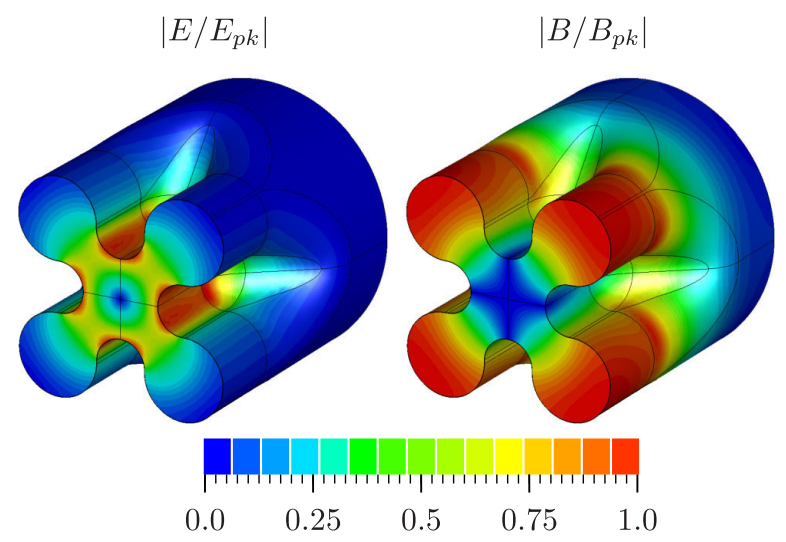

FIG. 12. Normalized field magnitude of the TE like quadrupole mode on the surface and in the transverse plane at the longitudinal center of a four-vane cavity. 
angle result in a cavity which is probably more difficult to fabricate and therefore excluded.

The transition from the quadrupolar contour to the circular beam pipe is defined by the tapering angle $\alpha_{\text {tap }}$. Note, the two curvatures along this tapering shown in Fig. 11(b) must not be smaller than the curvature provided by the iris ellipse in order to avoid additional field enhancement at the taper.

\section{A. Optimization}

For the four-vane cavity, not the magnetic but the electric field at the surface is the limiting factor. Correspondingly, we modify the prime objective such that $b_{2} / E_{p k}$ has to be maximized in the frame of the optimization. In contrast to the axially symmetrical geometries, the optimization of the four-vane shape relies on 3D eigenmode solvers using HFSS or CST MWS. Regardless, the simulation time for a cavity at $800 \mathrm{MHz}$ is on average in the same order of magnitude as the eigenmode simulations require for the previously discussed elliptical shapes using SLANS2. The reason is the compactness of the four-vane structure. The diameter of the cavity is approximately three times smaller than in the case of elliptical cavities.

A parametric model has been developed for both, HFSS and CST with the difficulty of ensuring a correct construction for any reasonable ensemble of design parameters according to Fig. 11. The model is able to cover both cases that are depicted in Figs. 11(c) and 11(d). The evaluation of peak fields at the surface and the calculation of the quadrupolar strength $b_{2}$ are post processing steps which can be defined in both programs either by using intrinsic functions or by self-written macros using for example VBSCRIPT. It turned out that the peak fields provided by HFSS are too unreliable such that we finally focused on CST.

Currently there is no build-in optimizer available in CST that incorporates a side condition to ensure a given frequency by adjusting one of the parameters. Therefore we consider the equator radius $r_{\text {eq }}$ likewise as a free variable and proceed the optimization in the following steps: 1 . The iris radius $r_{\text {ir }}$ and the tapering angle $\alpha_{\text {tap }}$ are fixed for the optimization. 2. Parameter sweeps are carried out with respect to $a_{\mathrm{ir}}, b_{\mathrm{ir}}, \alpha, l_{\mathrm{hv}}$, and $r_{\mathrm{eq}}$, providing the quadrupolar strength and peak fields at the surface. 3 . For each ensemble $\left(a_{\mathrm{ir}}, b_{\mathrm{ir}}, \alpha, l_{\mathrm{hv}}\right)$, evaluate the equator radius $r_{\mathrm{eq}}$ that satisfy the given frequency by interpolation. 4 . For each ensemble $\left(a_{\mathrm{ir}}, b_{\mathrm{ir}}, \alpha\right)$ evaluate the parameter $l_{\mathrm{hv}}$ (half of a vane length) that maximize $b_{2}$ with respect to the limiting field. As in the previous step, this is done by interpolation. 5. Analyze the dependency of $b_{2} / E_{p k}$ and $b_{2} / B_{p k}$ on the remaining free parameters $\left(a_{\mathrm{ir}}, b_{\mathrm{ir}}, \alpha\right)$ The optimization is carried out for various combinations of the aperture (iris radius $r_{\text {ir }}$ ) and the tapering angle $\alpha_{\text {tap }}$ as both parameters are expected to have a significant influence on the longitudinal and transverse impedance. Note, the center and half axes of the equator ellipse (with $a_{\mathrm{eq}}=b_{\mathrm{eq}}$ ) are defined by the other parameters such that $a_{\mathrm{eq}}$ is maximized. The number of simulations that have been done is in the order of 2000-5000 per iris radius and tapering angle and required between 12-30 hours.

\section{B. Optimal design parameters}

A total of 15 cavities operating at $800 \mathrm{MHz}$ have been optimized in terms of quadrupolar strength providing an iris radius of 20,30, and $40 \mathrm{~mm}$ and tapering angles between $30 \mathrm{deg}$ and $80 \mathrm{deg}$. The parameters and properties are summarized in the Supplemental Material at [23]. In the line of optimizations, it turned out that the maximum achievable quadrupolar strength reduces the larger the tangential angle between the iris and equator ellipses $\alpha$ is, similar to what is shown in Fig. 8(a). Therefore the angle was fixed to one degree.

The optimal cavity length including the taper varies from design to design in a range between $l_{\text {cav }}=200-300 \mathrm{~mm}$, hence, all of them are considerably above half the wave length.

Figure 13 shows an example of quadrupolar strength relative to the surface peak fields as a function of the remaining free parameters given by the iris ellipse. It can be seen that the ratio $B_{p k} / E_{p k}$ is always smaller than $2.13 \mathrm{mT} /(\mathrm{MV} / \mathrm{m})$ indicating the limitation by the electric field. The maximum in Fig. 13(b) yield an ellipse very close to a circle with $a_{\mathrm{ir}}<b_{\mathrm{ir}}$. Other apertures and tapering angles provided qualitatively the same result of having

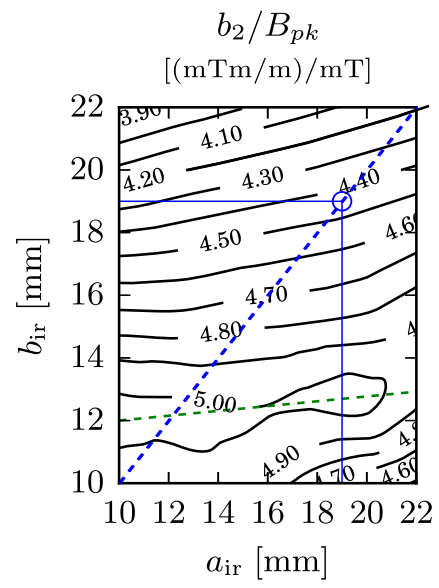

(a)

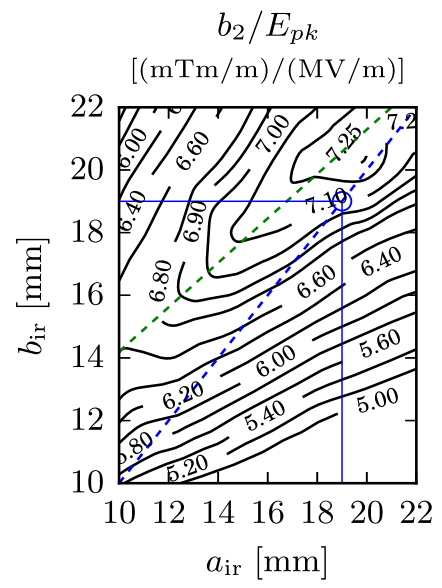

(b)
FIG. 13. Quadrupolar strength with respect to the magnetic (a) and electric (b) surface peak field as a function of the two half axes of the iris ellipse. In dashed blue, the reference line of a circular profile. In dashed green, regression line of the maximums in $\left(a_{\mathrm{ir}}, b_{\mathrm{ir}}\right)$. The cavity length is optimized for each data set with an iris radius $r_{\mathrm{ir}}=30 \mathrm{~mm}$, a tapering angle $\alpha_{\mathrm{tap}}=30 \mathrm{deg}$, and a tangential angle $\alpha=1 \mathrm{deg}$. The circle emphasizes the optimized design. 
almost a circular arc. As the maximum appears relatively flat, we decided to simplify the iris ellipse, in general, to a circle $\left(a_{\mathrm{ir}}=b_{\text {ir }}\right)$. The flatness allows large tolerances for the iris ellipse which facilitate the fabrication.

We did not choose the iris ellipse that maximizes $b_{2} / E_{p k}$. Rather, we applied a weighting between both $b_{2} / E_{p k}$ and $b_{2} / B_{p k}$ according to:

$$
\begin{aligned}
\frac{E_{0}}{b_{2}}\left(\frac{B_{p k}}{B_{0}}+\frac{E_{p k}}{E_{0}}\right) & =\frac{E_{0}}{B_{0}} \cdot \frac{B_{p k}}{b_{2}}+1 \cdot \frac{E_{p k}}{b_{2}} \\
& =w_{1} \cdot \frac{B_{p k}}{b_{2}}+w_{2} \cdot \frac{E_{p k}}{b_{2}},
\end{aligned}
$$

with $w_{1}<w_{2}$. This expression used as a goal function that has to be minimized lead to the optimum shown in Fig. 13 by the circles. The advantage of such a weighting is a slightly smaller iris ellipse without reducing the quadrupolar strength, appreciably. Note, the larger $b_{\text {ir }}$ the smaller the gap between the vanes which complicates the surface preparation of the cavity. Moreover the ratio of the surface peak fields is somewhat improved by this weighting as it includes the magnetic peak field as well.

\section{Properties of the optimized cavities}

The properties of the designs are summarized in Fig. 14. In agreement with the elliptical cavities, the quadrupolar strength rises with decreasing aperture $r_{\text {ir }}$, however, the values are significantly larger. Depending on its aperture a four-vane cavity is able to provide between two to five times more strength than a non-reentrant elliptical cavity with an iris radius of $r_{\text {ir }}=60 \mathrm{~mm}$. This exceeds clearly the performance gain obtained by reentrant elliptical cavities.

The ratio of the surface peak fields is as expected below $2.13 \mathrm{mT} /(\mathrm{MV} / \mathrm{m})$ but in contrast to elliptical cavities the ratio is not independent of the aperture. This becomes clear if we consider the gap between the vanes. The smaller the iris radius the smaller is the distance between the vanes which provide an alternating polarization in the azimuthal direction similar to a quadrupole magnet. Hence, the electric field at the corresponding surfaces increases while the peak magnetic field remains unchanged. Both, the quadrupolar strength as well as the ratio of peak fields are not noticeably influenced by the tapering angle $\alpha_{\text {tap }}$.

The effective longitudinal impedance of an entire system of four-vane cavities to satisfy the requirements in Table I is by a factor between two to four smaller than an adequate system of elliptical cavities provides. The effective transverse impedance is moderately larger but it is minor with respect to the LHC impedance budget. Depending on the tapering angle the longitudinal impedance varies by a factor of almost two. With respect to the achievable quadrupolar strength, the effective longitudinal

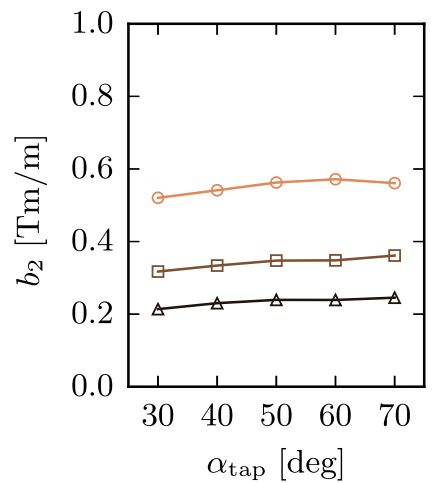

(a)

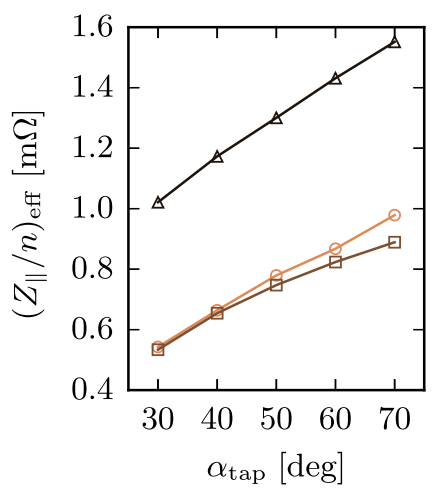

(c)

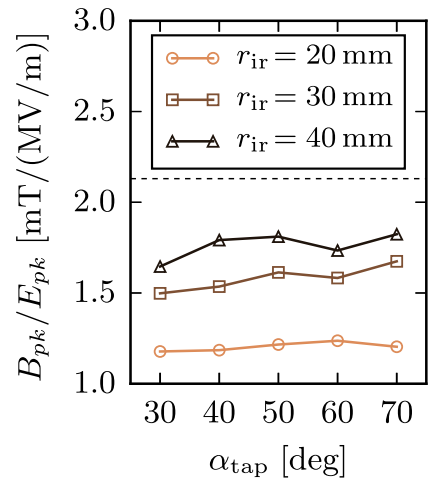

(b)

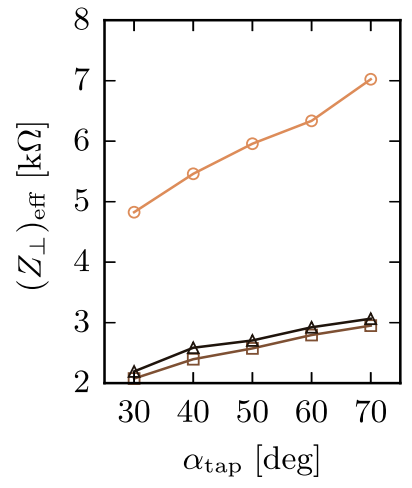

(d)
FIG. 14. Properties of the optimized $800 \mathrm{MHz}$ four-vane cavities with different iris radius $r_{\text {ir }}$ and tapering angle $\alpha_{\text {tap }}$. (a) Maximum achievable quadrupolar strength per cavity. (b) Ratio of surface peak fields. The effective longitudinal (c) and transverse (d) impedance are related to a whole system of cavities to provide the quadrupolar strength in Table I.

impedance of a system of four-vane cavities can go down to a tenth of the impedance of a corresponding system of elliptical cavities.

To conclude, by comparing all properties of the optimized designs, one may chose for HL-LHC a $800 \mathrm{MHz}$ four-vane cavity with an iris radius of $r_{\text {ir }}=30 \mathrm{~mm}$ and a tapering angle of $\alpha_{\text {tap }}=30 \mathrm{deg}$. Only one cavity is required to provide the value in Table I. The system provide an effective longitudinal impedance of $0.55 \mathrm{~m} \Omega$, almost eight times lower than the selected system of elliptical cavities in Sec. III C.

\section{LOMs and HOMs}

The large apertures at the ends of the cavity yield that only a few modes are trapped inside the cavity. Basically only the first two dipole modes are of interest: a $\mathrm{TE}_{111}$-like mode with a frequency between 700 and $750 \mathrm{MHz}$ and a $\mathrm{TM}_{111}$-like mode at around $1 \mathrm{GHz}$. With a bunch sigma of $80 \mathrm{~mm}$, the transverse wake spectrum is largely dominated by the first dipole mode which can be easily extracted if the beam pipe radius becomes slightly larger by $20-30 \mathrm{~mm}$. A corresponding sketch is illustrated in Fig. 15. The beam 


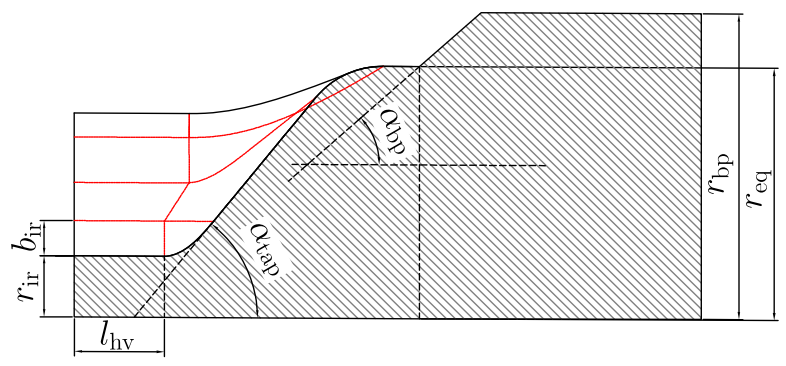

FIG. 15. Quarter of the longitudinal cross section of the fourvane cavity with beam pipe enhancement to allow the propagation of the first dipole mode.

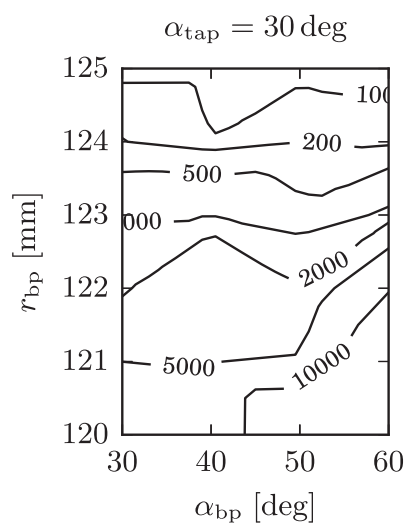

(a)

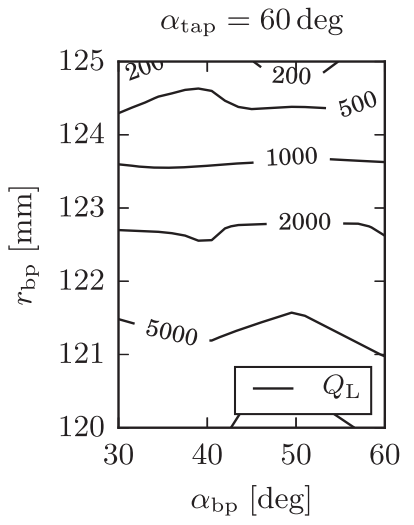

(b)
FIG. 16. Q-factor derived from the transverse wakefield for a design with an iris radius of $r_{\mathrm{ir}}=30 \mathrm{~mm}$ and a tapering angle $\alpha_{\text {tap }}$ of $30 \mathrm{deg}$ (a) or $60 \mathrm{deg}$ (b), respectively. The beam pipe radius $r_{\mathrm{bp}}$ varies in a range where the dominating dipole mode starts to propagate. The tapering angle $\alpha_{\mathrm{bp}}$ describing the beam pipe enhancement is applied on the axis of abscissae.

pipe diameter increase is defined by the final beam pipe radius $r_{\mathrm{bp}}$ and by a further taper with the angle $\alpha_{\mathrm{bp}}$. In Fig. 16, the quality factor derived from the transverse wakefield is shown for two different cavities as a function of the parameters $r_{\mathrm{bp}}$ and $\alpha_{\mathrm{bp}}$. Between a beam pipe radius of 120-125 $\mathrm{mm}$ the quality factor drops down as the $\mathrm{TE}_{11}$ mode starts to propagate through the beam pipe. Apparently, the tapering angles $\alpha_{\text {tap }}$ and $\alpha_{\mathrm{bp}}$ have a minor influence on the propagation.

In this example, the transverse wake field for a beam pipe radius of $r_{\mathrm{bp}}=124 \mathrm{~mm}$ or larger does not show any trapped modes. Based on this modification it is reasonable to apply LOM and HOM damping outside of the cryomodule using lossy materials in the beam pipe rather than couplers close to the cavity.

\section{E. Multipole field errors}

These errors mainly arise from higher order multipoles $b_{m}$ which satisfy the azimuthal periodicity of the
TABLE IV. Different multipoles of the four-vane cavity with $r_{\mathrm{ir}}=30 \mathrm{~mm}$ and $\alpha_{\mathrm{tap}}=30 \mathrm{deg}$.

\begin{tabular}{lcc}
\hline \hline Multipole & Unit & Value for $E_{p k}=50 \mathrm{MV} / \mathrm{m}$ \\
\hline$b_{2}$ & {$[\mathrm{Tm} / \mathrm{m}]$} & $3.47 \times 10^{-1}$ \\
$b_{6}$ & {$\left[\mathrm{Tm}^{5} / \mathrm{m}\right]$} & $9.62 \times 10^{4}$ \\
$b_{10}$ & {$\left[\mathrm{Tm}^{9} / \mathrm{m}\right]$} & $1.98 \times 10^{10}$ \\
\hline \hline
\end{tabular}

fundamental multipole $b_{n}$. They are correlated via the following condition:

$$
\frac{m}{n}=3,5,7, \ldots
$$

Further information can be found in [9]. As for the fundamental multipole $b_{2}$ describing the quadrupolar strength we use (2a) or (2b) to calculate the multipoles of higher order from an eigenmode solution. Table IV shows the result of one optimized cavity.

The elliptical cavity do not provide any higher order multipoles due to the exact sinusoidal azimuthal dependency of the field components which is not the case for the four-vane cavity. However, there may appear so-called random multipole errors due to fabrication errors and tolerances which is beyond the scope of this paper.

\section{RF-POWER}

Using the lumped circuit model from [11] one can derive a relation between the required input power $P_{\mathrm{g}}$ in order to compensate the beam loading and the coupling factor between the quadrupole resonator and the input coupler represented by the external quality factor $Q_{\text {ext }}$. According to [11], the input power is given by:

$$
P_{\mathrm{g}}=\frac{1}{2}(R / Q)_{\|}\left|I_{\mathrm{g}}\right|^{2}
$$

Note, we apply the circuit definition of the $R / Q$ values according to:

$$
(R / Q)_{\|}=\frac{V_{\|}^{2}}{2 \omega U},
$$

with longitudinal voltage $V_{\|}$, the stored energy $U$ inside the cavity and $\omega$ as the angular frequency of the rf field. The model current $I_{\mathrm{g}}$ of the lumped circuit model, is calculated by:

$$
\begin{aligned}
I_{\mathrm{g}}= & {\left[\frac{V_{\|}}{2(R / Q)_{\|}}\left(\frac{1}{Q_{\mathrm{ext}}}+\frac{1}{Q_{0}}\right)+I_{\mathrm{b}, \mathrm{DC}} F_{\mathrm{b}} \sin \phi\right] } \\
& +j\left[I_{\mathrm{b}, \mathrm{DC}} F_{\mathrm{b}} \cos \phi-\frac{V_{\|} \Delta \omega}{\omega(R / Q)_{\|}}\right],
\end{aligned}
$$

with the intrinsic quality factor $Q_{0}$ of the cavity, the adequate DC beam current $I_{\mathrm{b}, \mathrm{DC}}$ weighted by the 
bunch form factor $F_{\mathrm{b}}$, the synchronous phase angle $\phi$, and the angular frequency deviation $\Delta \omega$ between cavity resonance and the provided wave from the power coupler.

If we assume $Q_{0} \gg Q_{\text {ext }}$, a correct tuning of the cavity such that $\Delta \omega=0$ and synchronous phase of the longitudinal voltage $\phi=0$ which means that the deflective voltage $V_{\perp}$ is on crest as foreseen for the quadrupole resonator operation, Eqs. (11) and (13) simplify to:

$$
P_{\mathrm{g}}=\frac{V_{\|}^{2}}{8(R / Q)_{\|} Q_{\mathrm{ext}}}+\frac{1}{2}(R / Q)_{\|} Q_{\mathrm{ext}} I_{\mathrm{b}, \mathrm{DC}}^{2} F_{\mathrm{b}}^{2}
$$

In order to investigate the worst case we consider the azimuthal angle $\varphi$ where the longitudinal voltage $V_{\|}$is maximal for a given radius $\rho_{0}$. For the elliptical cavity, this voltage can be derived exactly from (4) based on the quadrupolar strength. In case of the four-vane cavity, Eq. (4) provides an approximation with the correction factors related to the multipoles described in Table IV. In general, this voltage is calculated by the eigenmode solution for the individual cavity. As $V_{\|}$is proportional to the squared distance of the beam from the center $\rho$, the longitudinal $R / Q$ ratio is proportional to $\rho^{4}$. Correspondingly in (14), the first term is independent of the beam displacement whereas the second term, which is related to beam loading effect, is proportional to the fourth power of the displacement. Figure 17 shows the required input power for an optimized four-vane cavity as a function of the external quality factor for different transverse positions of the bunch. The needed input power is very moderate and by almost one order of magnitude smaller than required for the HL-LHC crab cavities [24], even for a bunch position as much as few $\mathrm{mm}$ from the cavity axis. This is only true if $Q_{\text {ext }}>1 \times 10^{6}$ can be used in operating the cavity.

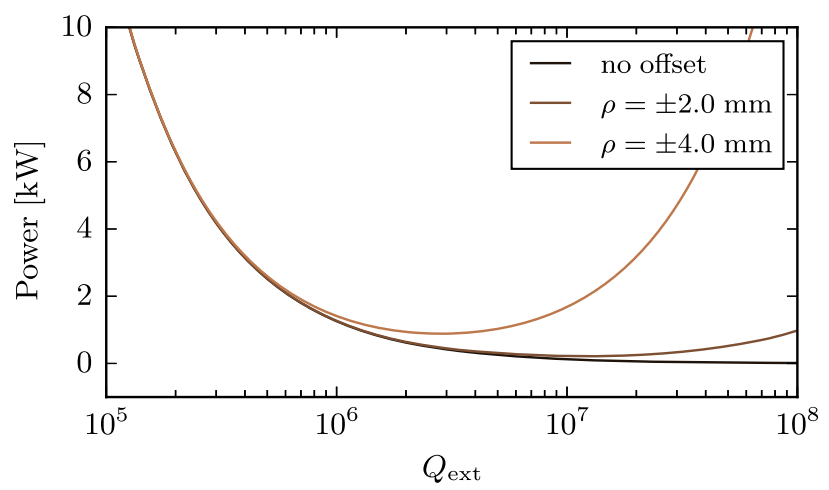

FIG. 17. Input power as a function of the external quality factor for a four-vane cavity with an iris radius of $r_{\text {ir }}=30 \mathrm{~mm}$ and a tapering angle of $\alpha_{\text {tap }}=30 \mathrm{deg}$. The beam offset is represented by the radial coordinate $\rho$. The bunch form factor $F_{\mathrm{b}}$ at $800 \mathrm{MHz}$ is around 0.5 [11].

\section{CONCLUSION}

Two methods have been presented to optimize the elliptical and the four-vane quadrupole resonator by means of their quadrupolar strength with respect to the limiting surface peak fields. In principle, these methods can be applied to any arbitrary geometry for either axially symmetrical or axially nonsymmetrical cavities. Considering the maximum achievable quadrupolar strength of a non-reentrant elliptical cavity as a reference, the reentrant elliptical cavity is able to provide up to $30 \%$ more strength. The four-vane cavity can deliver between two to five times more strength than the reference depending on its iris radius. It should be noted that this is only true for small iris radii $r_{\text {ir }}<50 \mathrm{~mm}$. Otherwise, elliptical cavities become more efficient.

The significantly lower longitudinal impedance, the sparse LOM and HOM spectrum, and the lower number of required cavities argue clearly for the four-vane cavity if small apertures are allowed. The compactness of the cavity is a further advantage with respect to the size of the cryomodule but complicates certainly the fabrication and surface cleaning. Though it was shown that the performance of the four-vane quadrupole resonator is marginally influenced by deviations in the iris ellipse. Hence, the difficulty lies rather in a proper surface preparation impacting the maximum achievable peak field. We therefore favor a design not with the smallest investigated aperture but the optimized geometry with an iris radius of $r_{\text {ir }}=30 \mathrm{~mm}$ and the tapering angle of $\alpha_{\text {tap }}=30 \mathrm{deg}$. For this example, we have calculated in Sec. IV E systematic multipole errors which serve as input for eventual beam dynamic simulations in the future. Likewise for this example, the required rf power lies significantly below $10 \mathrm{~kW}$ with an optimal external quality factor in the order of $Q_{\text {ext }}=3 \times 10^{6}$. Hence, the rf power needed is very moderate which facilitates the requirements for the power coupler and the whole rf system.

\section{ACKNOWLEDGMENTS}

We especially acknowledge the fruitful discussions with Michael Schenk and Kevin Lee. The authors would also like to thank Rama Calaga, Slava Yakovlev, and Shahnam Gorgi Zadeh for their helpful recommendations.

[1] L. D. Landau, Zh. Eksp. Teor. Fiz. 16, 574 (1946) [On the vibrations of the electronic plasma, J. Phys. USSR 10, 25 (1946)].

[2] A. Chao, Physics of Collective Beam Instabilities in High Energy Accelerators (Wiley, New York, 1993), Chap. Landau Damping, p. 219.

[3] E. Métral, B. Salvant, and N. Mounet, Technical Report No. CERN-ATS-2011-102, 2011. 
[4] J. Gareyte, J.-P. Koutchouk, and F. Ruggiero, Technical Report No. CERN-LHC-Project-Report-91, 1997.

[5] E. Métral, G. Arduini, L. Barranco Navarro, X. Buffat et al., Technical Report No. CERN-ACC-2016-0098, 2016.

[6] A. Grudiev, Radio frequency quadrupole for Landau damping in accelerators, Phys. Rev. ST Accel. Beams 17, 011001 (2014).

[7] A. Grudiev and M. Schenk, Radio frequency quadrupole for landau damping in accelerators. Analytical and numerical studies, in Proceedings of HB2014 (JACoW, East Lansing, MI, USA, 2014), p. 315.

[8] L. R. Carver, M. Schenk, R. De Maria, K. S. B. Li et al., Technical Report No. CERN-ACC-NOTE-2017-0012, 2017.

[9] A. Wolski, Beam Dynamics in High Energy Particle Accelerators (Imperial College Press, London, 2014), p. 26.

[10] J. Barranco García, R. De Maria, A. Grudiev, R. Tomás García, R. B. Appleby et al., Long term dynamics of the high luminosity Large Hadron Collider with crab cavities, Phys. Rev. Accel. Beams 19, 101003 (2016).

[11] J. Tückmantel, Technical Report No. CERN-ATS-Note2011-002 TECH, 2010.

[12] M. Schenk, A. Grudiev, K. S. B. Li, and K. Papke, Technical Report No. CERN-ACC-2016-0096, 2016.

[13] R. Wanzenberg, Technical Report No. DESY-TESLA2001-33, 2001.

[14] B. Aune, R. Bandelmann, D. Bloess, B. Bonin et al., Superconducting TESLA cavities, Phys. Rev. ST Accel. Beams 3, 092001 (2000).
[15] S. U. De Silva and J. R. Delayen, Design evolution and properties of superconducting parallel-bar rf-dipole deflecting and crabbing cavities, Phys. Rev. ST Accel. Beams 16, 012004 (2013).

[16] V. Shemelin, Optimal choice of cell geometry for a multicell superconducting cavity, Phys. Rev. ST Accel. Beams 12, 114701 (2009).

[17] F. Ruggiero, Technical Report No. CERN-SL-95-09-AP, 1995.

[18] Y. H. Chin, Advances and applications of ABCI, in Proceedings of International Conference on Particle Accelerators (IEEE, Washington, DC, USA, 1993), Vol. 5, p. 3414.

[19] I. Zagorodnov, K. L. F. Bane, and G. Stupakov, Calculation of wakefields in 2D rectangular structures, Phys. Rev. ST Accel. Beams 18, 104401 (2015).

[20] CST-Computer Simulation Technology Ver. 2016 (CST AG, Darmstadt, Germany, 2016).

[21] D. G. Myakishev and V. P. Yakovlev, The new possibilities of SuperLANS code for evaluation of axisymmetric cavities, in Proceedings Particle Accelerator Conference (IEEE, Dallas, TX, USA, 1995), Vol. 4, p. 2348.

[22] HFSS_High Frequency Structural Simulator Ver. 2014 (ANSYS Inc., Canonsburg, Pennsylvania, USA, 2014).

[23] See Supplemental Material at http://link.aps.org/ supplemental/10.1103/PhysRevAccelBeams.20.082001 for optimized design parameters of rf quadrupole resonators for Landau damping.

[24] R. Calaga, Crab Cavities for the LHC Upgrade, in Workshop on LHC Performance, Chamonix (CERN, Chamonix, 2012). 